\begin{abstract}
${ }^{1}$ Dermatólogo, Servicio de Dermatología Hospital Barros Luco Trudeau, Santiago, Chile. ${ }^{2}$ Residente de Dermatología, Departamento de Dermatología, Hospital Clínico Universidad de Chile, Santiago, Chile ${ }^{3}$ Medical Resident Officer, Royal Perth Hospital, Australia.

Trabajo no recibió financiamiento. Los autores declaran no tener conflictos de interés.

Recibido el 8 de julio de 2019, aceptado el 4 de noviembre de 2019 .

Correspondencia: Daniela Soledad Carvajal Aguilera E-mail: dscarvajal@ gmail.com

\section{Resumen}

El poroma es un tumor benigno infrecuente derivado de la porción intraepitelial del epitelio ductal de la glándula sudorípara. La presentación clínica más habitual es un pápula o nódulo de color rosado a rojo, de pequeño diámetro, localizado con mayor frecuencia en palmas y plantas, generalmente asintomático. Debido a su variabilidad morfológica, los poromas son usualmente dificiles de reconocer, siendo la dermatoscopía una herramienta útil en su diagnóstico. Presentamos el caso de un paciente masculino con un gran tumor en su pierna derecha de $40 \mathrm{~mm}$ de diámetro, con características dermatoscópicas compatibles con poroma no pigmentado. El diagnóstico confirmatorio se realizó con una biopsia incisional seguido de la extirpación quirúrgica completa del tumor.
\end{abstract}

Palabras claves: poroma; dermatoscopía; patrón vascular polimorfo; histopatología.

\title{
Poroma no pigmentado inusualmente grande: reporte de un caso y revisión de la literatura
}

Miguel Espinoza ${ }^{1}$, Daniela Carvajal ${ }^{2}$, Francisco Bobadilla ${ }^{1}$, Andrea Zamudio ${ }^{3}$

\section{SUMMARY}

Poroma is a rare benign tumour derived from the intraepithelial portion of the sweat glands' ductal epithelium. The most common clinical presentation is a pink to red papule or nodule, small in diameter, predominantly in the palms and soles and generally asymptomatic. Due to its morphologic variability, poromas are usually difficult to recognize, thus dermatoscopy becomes a useful aid in its diagnosis. We present the case of a male patient with a large tumour on his right leg, 40 $\mathrm{mm}$ in diameter, with dermatoscopic features consistent with non-pigmented poroma. Confirmatory diagnosis was made by incisional biopsy followed by the total surgical excision of the tumor.

Key words: poroma; dermatoscopy; polymorphic vascular pattern; histopathology
$\mathrm{E}$ 1 poroma corresponde a una neoplasia benigna infrecuente originada del epitelio ductal intraepidérmico de la glándula sudorípara. Con frecuencia se presenta en palmas y plantas como una pápula o nódulo no pigmentado, bien delimitado, de pequeño diámetro, generalmente asintomático ${ }^{1}$. Los poromas pueden presentarse de forma infrecuente como nódulos pigmentados en áreas no acrales simulando otros tumores cutáneos². La dermatoscopía es una herramienta no invasiva especialmente útil en casos de poromas de presentación atípica ${ }^{1}$.

Presentamos el caso de un paciente de sexo masculino con un tumor en su pierna derecha de $40 \mathrm{~mm}$ de diámetro, con características dermatoscópicas compatibles con poroma no pigmentado. El análisis histológico de la totalidad de la lesión confirmó el diagnóstico.

\section{Caso Glínico}

Paciente de sexo masculino, 62 años, con antecedentes de hipertensión arterial y diabetes mellitus tipo II. Consulta por un tumor en pierna derecha de 25 años de evolución, de crecimiento lento, asociado a dolor y sangrado en relación a trauma local. Al examen físico se observó en cara posterior de pierna derecha un tumor exofítico de $40 \times 40 \mathrm{~mm}$, de color rojo pálido, de consistencia firme, con focos de erosión y hemorragia (Figura 1). La dermatoscopía mostró áreas desestructuradas amarillas, hemorragias focales y áreas de queratinización (Figura 2A). El análisis de estructuras vasculares mostró un patrón vascular polimorfo, con vasos en forma de cáliz, vasos tipo hoja, vasos lineales irregulares y vasos en horquilla, rodeados de un halo de color blanco. Agregados tipo huevo de rana y áreas rojo lechosas también fueron 


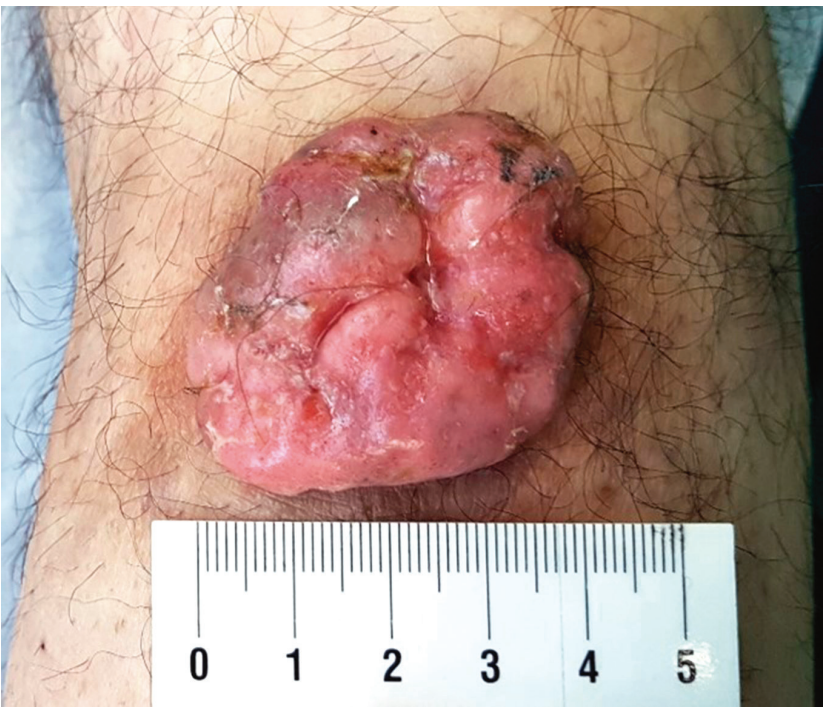

Figura 1

Paciente con tumor exofitico en cara posterior de pierna derecha de 40 x 40 mm de diámetro.

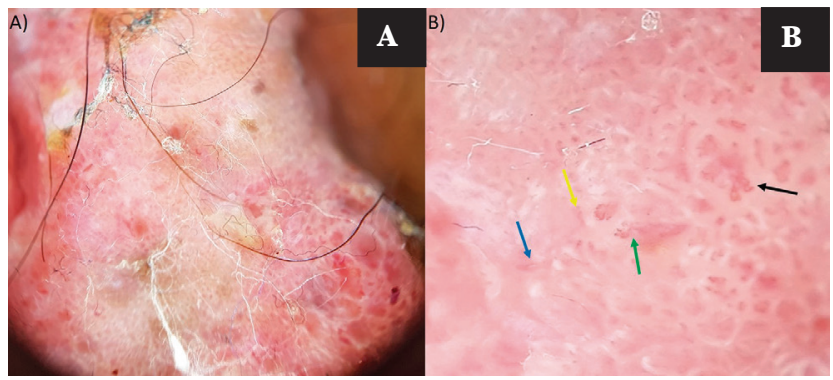

Figura 2

Características dermatoscópicas de poroma no pigmentado.

A. Áreas amarillas desestructuradas, hemorragias focales, áreas queratinizantes y agregados en huevo de rana.

B. Patrón vascular polimorfo: vasos en forma de cáliz (flecha negra), vasos en forma de hoja (flecha verde), vasos lineales irregulares (flecha amarilla) y vasos en horquilla (flecha azul). observadas (Figura 2B). La biopsia incisional describió la presencia de un infiltrado tumoral de células poroides cuboideas, pequeñas y monomorfas, que se extendían desde la epidermis a la dermis. Con mayor aumento, se observaron células poroides con un núcleo redondo, basófilo y monomorfo, con escaso citoplasma eosinófilo (Figura 3). Estos hallazgos fueron compatibles con el diagnóstico de poroma, por lo que posteriormente se procedió a la extirpación completa del tumor. El análisis histológico de la totalidad de la lesión confirmó el diagnóstico.

\section{Discusión}

El poroma es un tumor anexial benigno, a menudo solitario, que afecta principalmente a pacientes entre la cuarta y sexta décadas de la vida, con un leve predominio en hombres ${ }^{2}$. Se presenta como una pápula o nódulo de color rosado o rojo claro, de consistencia firme, con predominio en plantas y palmas, que es donde se concentra la mayor cantidad de glándulas sudoríparas ecrinas, pudiendo desarrollarse en cualquier ubicación que presente glándulas sudoríparas ${ }^{3}$. Generalmente es asintomático, sin embargo, la presencia de dolor, sangrado o purito debe hacerse sospechar su transformación maligna ${ }^{4}$.

El poroma con frecuencia se presenta como un tumor de pequeño diámetro ${ }^{1}$. Un estudio multicéntrico que incluyó 113 poromas, determinó un diámetro promedio de $8,3 \mathrm{~mm}^{2}$, existiendo reportes aislados de poromas con tamaño mayor a $40 \mathrm{~mm}^{5}$. Los poromas pueden presentarse de forma infrecuente como

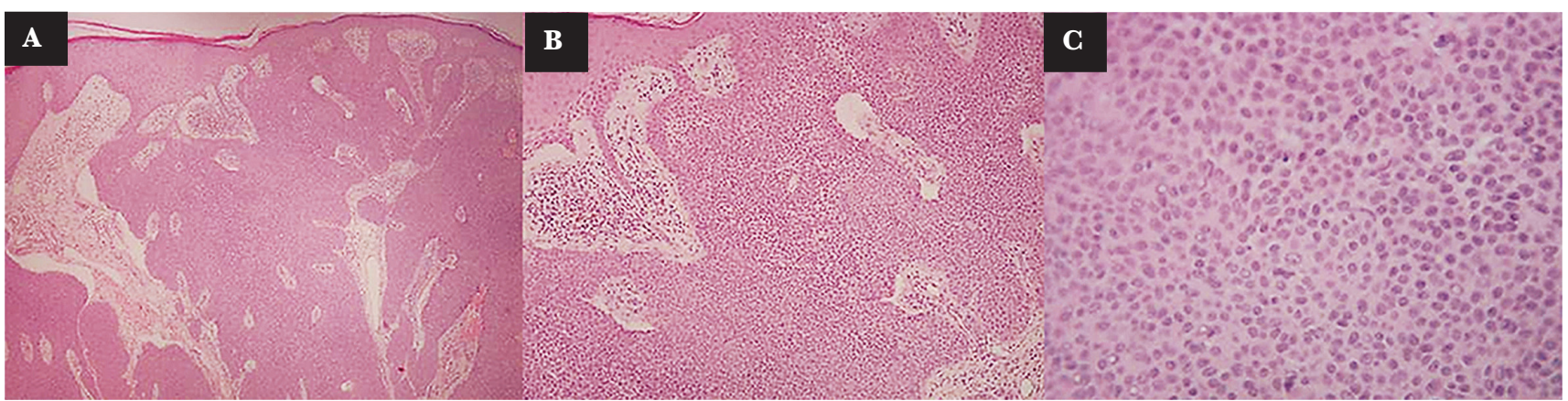

Figura 3

Sección de piel teñida con hematoxilina - eosina. A) 40x. Tumor infiltrativo formado por cordones anastomosantes compuestos por células poroides pequeñas y compactas, que se extienden desde la epidermis a la dermis. B) 100x. Cordones tumorales bien circunscritos dentro de la dermis formados por células poroides redondeadas. C) 400x. Células poroides con núcleo basófilo, redondo, monomorfo y con escaso citoplasma eosinófilo. 
nódulos pigmentados o tumores pedunculados en sitios no acrales, simulando otros tumores cutáneos como queratosis seborreica, granuloma telangiectásico, carcinoma basocelular, carcinoma espinocelular o melanoma amelanótico ${ }^{3}$.

Histológicamente, el poroma se presenta como un tumor bien delimitado, compuesto por células poroides cuboideas uniformes que se extienden desde la capa basal de la epidermis hasta la dermis ${ }^{3,6}$. Las células poroides se caracterizan por presentar un núcleo basófilo, redondeado y monomorfo, junto con un citoplasma compacto eosinófilo ${ }^{3,4}$. El poroma puede ser subdividido en base a la localización de las células poroides en relación a la epidermis, clasificándose en intraepidérmico, yuxtaepidérmico o intradérmico ${ }^{3,6}$. Tradicionalmente, se ha descrito que los poromas presentan un origen ecrino, sin embargo, existen reportes de poroma con diferenciación apocrina ${ }^{6}$.

La dermatoscopía es una herramienta no invasiva que resulta útil en el diagnóstico diferencial de neoplasias cutáneas. En el caso del poroma, su principal utilidad es en tumores no pigmentados, reportándose con frecuencia un patrón vascular polimorfo ${ }^{1}$. En poromas no pigmentados se ha descrito la presencia de vasos tipo flor (también conocidos como flor de cerezo), correspondientes a vasos arboriformes con terminaciones en forma de círculos pequeños, y vasos tipo hoja, conformados por un vaso central desde el cual emergen ramificaciones ${ }^{7}$. Otros hallazgos son la presencia de agregados en huevos de rana conformados por glóbulos o estructuras tipo laguna de color rosado a rojo separados por bandas en forma de red de color blanquecino, y vasos en forma de cáliz, correspondientes a vasos lineales irregulares con una terminación semielíptica ${ }^{1,8}$. Otras estructuras vasculares descritas incluyen vasos glomerulares, vasos en horquilla y vasos lineales irregulares, a menudo rodeados de un halo blanquecino, y áreas desestructuradas redondeadas u ovales de color blanco o rosado pálido ${ }^{1}$. Estas áreas desestructuradas y el halo perivascular se correlacionan con características histopatológicas específicas, como fibroplasia laminar dérmica y edema fibrinoide alrededor de los vasos dilatados, respectivamente ${ }^{9}$. Otros hallazgos dermatoscópicos descritos son áreas escamosas o de queratinización, zonas de erosión/ulceración y focos de hemorragia ${ }^{1,2}$.
Un estudio observacional multicéntrico ${ }^{2}$ publicado por Marchetti et al. incluyó un total de 113 poromas, de los cuales el 85,8\% correspondió a la variante no pigmentada. Este trabajo describió como hallazgos dermatoscópicos característicos la presencia de áreas blanquecinas entrecruzadas alrededor de los vasos sanguíneos, áreas amarillas desestructuradas, glóbulos rojo lechosos y vasos pobremente visualizados. La presencia de vasos arboriformes con terminaciones redondeadas se asoció a poroma, pero no alcanzó una significación estadística. Así, la presencia de cualquiera de estas cinco características se asoció con una sensibilidad y especificidad de $62,8 \%$ y $82 \%$, respectivamente, siendo concordante con lo reportado en estudios previos ${ }^{1,6}$.

El diagnóstico de poroma debe ser confirmado por el análisis histológico de la totalidad de la lesión, debido a que biopsias incisionales pueden resultar falsamente benignas. La extirpación quirúrgica completa con márgenes libres es el tratamiento de elección, debido al riesgo de recurrencias y a la posibilidad de una transformación maligna a porocarcinoma ${ }^{4}$.

\section{Conclusión}

A pesar que el diagnóstico debe ser confirmado por biopsia, herramientas no invasivas como la dermatoscopía pueden ayudar en el diagnóstico diferencial, siendo especialmente útil en casos de presentación atípica como la de nuestro paciente. Un alto nivel de sospecha clínica y la extirpación quirúrgica completa con biopsia son esenciales para establecer un diagnóstico oportuno y preciso.

Agradecimientos al servicio de Anatomía Patológica del Hospital Barros Luco Trudeau. 


\section{REFERENCIAS}

1. Espinosa AE, Ortega BG, Venegas RQ, Ramirez RG. Dermoscopy of non-pigmented eccrine poromas: study of Mexican cases. Dermatol Pract Concept 2013;3:25-8

2. Marchetti MA, Marino ML, Virmani P, Dusza SW, Marghoob AA, Nazzaro G, et al. Dermoscopic features and patterns of poromas: a multicentre observational case-control study conducted by the International Dermoscopy Society. J Eur Acad Dermatol Venereol 2018;32:1263-1271

3. Bombonato C, Piana S, Moscarella E, Lallas A, Argenziano G, Longo C. Pigmented eccrine poroma: dermoscopic and confocal features. Dermatol Pract Concept 2016;6:59-62

4. Abarzua A, Alvarez-Veliz S, Moll-Manzur C. Concomitant poroma and porocarcinoma. An Bras Dermatol 2017;92:550-552

5. Sakuma T, Itou Y, Shibata A. Large pedunculated poroma with apocrine differentiation. Int J Dermatol 2014;53:e234-6

6. Shalom A, Schein O, Landi C, Marghoob A, Carlos B, Scope A. Dermoscopic findings in biopsy-proven poromas. Dermatol Surg 2012;38:1091-6

7. Aydingoz IE. New dermoscopic vascular patterns in a case of eccrine poroma. J Eur Acad Dermatol Venereol 2009;23:725-6

8. Minagawa A, Koga H, Takahashi M, Sano K, Okuyama R. Dermoscopic features ofnonpigmented eccrine poromas in association with their histopathological features. Br J Dermatol 2010;163:1264-8

9. Ferrari A, Buccini P, Silipo V, De Simone P, Mariani G, Marenda S, et al. Eccrine poroma: a clinical-dermoscopic study of seven cases. Acta Derm Venereol 2009;89:160-4

10. Brugues A, Gamboa M, Alos L, Carrera C, Malvehy J, Puig S. The challenging diagnosis of eccrine poromas. J Am Acad Dermatol 2016;74:e113-5 